\title{
Running interfacial waves in two-layer fluid system subject to longitudinal vibrations
}

\author{
D. S. Goldobin,,${ }^{1,2,3}$ A. V. Pimenova, ${ }^{1}$ K. V. Kovalevskaya, ${ }^{1}$ D. V. Lyubimov, ${ }^{2}$ and T. P. Lyubimova ${ }^{1,2}$ \\ ${ }^{1}$ Institute of Continuous Media Mechanics, UB RAS, 1 Academik Korolev str., Perm 614013, Russia \\ ${ }^{2}$ Department of Theoretical Physics, Perm State University, 15 Bukireva str., Perm 614990, Russia \\ ${ }^{3}$ Department of Mathematics, University of Leicester, University Road, Leicester LE1 7RH, UK
}

\begin{abstract}
We study the waves at the interface between two thin horizontal layers of immiscible fluids subject to high-frequency horizontal vibrations. Previously, the variational principle for energy functional, which can be adopted for treatment of quasi-stationary states of free interface in fluid dynamical systems subject to vibrations, revealed existence of standing periodic waves and solitons in this system. However, this approach does not provide regular means for dealing with evolutionary problems: neither stability problems nor ones associated with propagating waves. In this work, we rigorously derive the evolution equations for long waves in the system, which turn out to be identical to the 'plus' (or 'good') Boussinesq equation. With these equations one can find all timeindependent-profile solitary waves (standing solitons are a specific case of these propagating waves), which exist below the linear instability threshold; the standing and slow solitons are always unstable while fast solitons are stable. Depending on initial perturbations, unstable solitons either grow in an explosive manner, which means layer rupture in a finite time, or falls apart into stable solitons. The results are derived within the long-wave approximation as the linear stability analysis for the flat-interface state [D.V. Lyubimov, A.A. Cherepanov, Fluid Dynamics 21, 849-854 (1987)] reveals the instabilities of thin layers to be long-wavelength.
\end{abstract}

PACS numbers: $\quad 47.35 . \mathrm{Fg}, 47.15 . \mathrm{gm}, 47.20 . \mathrm{Ma}$

\section{INTRODUCTION}

In 1, 2] Wolf reported experimental observations of the occurrence of steady wave patterns on the interface between immiscible fluids subject to horizontal vibrations. The build-up of the theoretical basis for these experimental findings was initiated with the linear instability analysis of the flat state of the interface [3 [5] (see Fig.[1] for the sketch of the system considered in these works). Specifically, it was found that in thin layers the instability is a long-wavelength one [3]. In [4, 5], the linear stability was determined for the case of arbitrary frequency of vibrations.

In spite of the substantial advance in theoretical studies, the problem proved to require subtle approaches; a comprehensive straightforward weakly-nonlinear analysis of the system subject to high-frequency vibrations still remains lacking in the literature (as well as the long-wavelength one). The approach employed in [3] can be (and was) used for analysis of time-independent quasi-steady patterns (including non-linear ones) only, but not the evolution of these patterns over time. This "restricted" analysis of the system revealed that quasisteady patterns can occur both via sub- and supercritical pitchfork bifurcations, depending on the system parameters. Later on, specifically for thin layers, which will be the focus of our work, the excitation of patterns was shown to be always subcritical [6] (paper [6] is published only in Russian, although the result can be derived from [3] as well). Within the approach of [3, 6] neither time-dependent patterns nor the stability of timeindependent patterns can be analyzed. Specifically for the case of subcritical excitation, time-independent patterns may belong to the stability boundary between the attraction basins of the flat-interface state and the finiteamplitude pattern state in the phase space. 24]

In this work we accomplish the task of derivation of the governing equations for dynamics of patterns on the interface of two-layer fluid system within the approximation of inviscid fluids. In Wolf's experiments [1, 2], the viscous boundary layer in the most viscous liquid was an order of magnitude thinner than the liquid layer, meaning the approximation of inviscid liquid is relevant. The layer is assumed to be thin enough for the evolving patterns to be long-wavelength [3]. With the governing equations we analyze the dynamics of the system below the linear instability threshold, where the system turns out to be identical to the 'plus' Boussinesq equation. The system admits soliton solutions, these solutions are parameterized with single parameter, soliton speed. The maximal speed of solitons equals the minimal group velocity of linear waves in the system; the soliton waves move always slower than the packages of linear waves. Stability analysis reveals that the standing and slow solitons are unstable while fast solitons are stable. The system, as the 'plus' Boussinesq equation, is known to be fully integrable.

Recently, the problem of stability of a liquid film on a horizontal substrate subject to tangential vibrations was addressed in the literature [7]. The stability analysis for space-periodic patterns and solitary waves for the latter system was reported in [8]. The similarity of this problem with the problem we consider and expected similarity of results are illusive. Firstly, for the problem of [7] the liquid film is involved into oscillating motion only due to viscosity, an inviscid liquid will be motionless over the tangentially vibrating substrate, while in the system we consider the inviscid fluid layers will oscillate due to mo- 


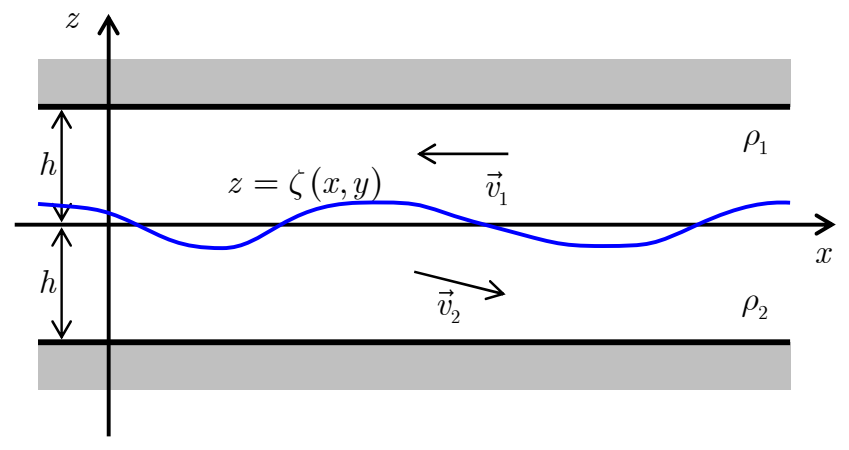

FIG. 1: Sketch of a two-layer fluid system subject to longitudinal vibrations and the coordinate frame.

tion of the lateral boundaries of the container and fluid incompressibility [3 5]. Secondly, the single-film case corresponds to the case of zero density of the upper layer in a two-layer system; in the system we consider this is a very specific case. These dissimilarities have their reflection in the resulting mathematical models; the governing equations for long-wavelength patterns derived in [7] are of the 1st order with respect to time and the 4th order with respect to the space coordinate and describes purely dissipative patterns in the viscous fluid, while the equation we will report is of 2 nd order in time, 4 th order in space and describes non-dissipative dynamics.

The paper is organized as follows. In Sec.II we provide a physical description and mathematical model for the system under consideration. In Sec.[II the governing equations for long-wavelength patterns are derived and discussed. In Sec.[IV soliton solutions are presented and their stability properties are analyzed. Conclusions are drawn in Sec.V.

\section{PROBLEM STATEMENT AND GOVERNING EQUATIONS}

We consider a system of two horizontal layers of immiscible inviscid fluid, confined between two impermeable horizontal boundaries (see Fig.11). The system is subject to high-frequency longitudinal vibrations of linear polarization; the velocity of vibrational motion of the system is be $e^{i \omega t}+c . c$. (here "c.c." stands for complex conjugate). For simplicity, we consider the case of equal thickness, say $h$, of two layers, which is not expected to change the qualitative picture of the system behavior [25] but makes calculations simpler. The density of upper liquid $\rho_{1}$ is smaller than the density of the lower one $\rho_{2}$. We choose the horizontal coordinate $x$ along the direction of vibrations, the $z$-axis is vertical with origin at the unperturbed interface between layers.

In this system, at the limit of infinitely extensive layers, the state with flat interface $z=\zeta(x, y)=0$ is always possible. In real layers of finite extent, the oscillating lateral boundaries enforce liquid waves perturbing the interface; however, at a distance from these boundaries the inter- face will be nearly flat as well. For inviscid fluids, this state (the ground state) features spatially homogeneous pulsating velocity fields $\vec{v}_{j 0}$ in both layers;

$$
\begin{gathered}
\vec{v}_{j 0}=a_{j}(t) \vec{e}_{x}, \quad a_{j}(t)=A_{j} e^{i \omega t}+c . c ., \\
A_{1}=\frac{\rho_{2} b}{\rho_{1}+\rho_{2}}, \quad A_{2}=\frac{\rho_{1} b}{\rho_{1}+\rho_{2}},
\end{gathered}
$$

where $j=1,2$ and $\vec{e}_{x}$ is the unit vector of the $x$-axis. All equations and parameters in this subsection are dimensional. The time instant $t=0$ is chosen so that $b$ and $A_{j}$ are real. The result (10) follows from the condition of zero pressure jump across the uninflected interface and the condition of the total fluid flux through the vertical crosssection being equal $\int_{-h}^{+h} v^{(x)} d z=2 h\left(b e^{i \omega t}+\right.$ c.c.) (which is due to the system motion with velocity $b e^{i \omega t}+$ c.c.).

Considering the flow of inviscid fluid, it is convenient to introduce the potential $\phi_{j}$ of the velocity field;

$$
\vec{v}_{j}=-\nabla \phi_{j} .
$$

The mass conservation law for incompressible fluid, $\nabla$. $\vec{v}_{j}=0$, yields the Laplace equation for the potential, $\Delta \phi_{j}=0$. The kinematic conditions on the top and bottom boundaries

$$
\phi_{1 z}(z=h)=\phi_{2 z}(z=-h)=0
$$

and on the interface $z=\zeta(x, y)$

$$
\begin{aligned}
& \dot{\zeta}=-\phi_{1 z}+\nabla \phi_{1} \cdot \nabla \zeta, \\
& \dot{\zeta}=-\phi_{2 z}+\nabla \phi_{2} \cdot \nabla \zeta
\end{aligned}
$$

are also to be taken into account. (In what follows, the upper dot stands for the time-derivative and letter in subscript denotes partial derivative with respect to the corresponding coordinate.) Equations (4) and (5) can be derived from the condition that the points of zero value of the distance function $F=z-\zeta(x, y)$, which correspond to the position of the interface, move with fluid, i.e., the Lagrangian derivative (material derivative) $d F / d t=\partial F / \partial t+\vec{v} \cdot \nabla F$ is zero on the interface: $-\dot{\zeta}+$ $v^{(z)}-\vec{v} \cdot \nabla \zeta=0$, and this holds for both fluids.

After substitution of the potential flow, the Euler equation takes the following form:

$$
\nabla\left(-\dot{\phi}_{j}+\frac{1}{2}\left(\nabla \phi_{j}\right)^{2}\right)=\nabla\left(-\frac{1}{\rho_{j}} p_{j}-g z\right)
$$

where $g$ is the gravity. The latter equation provides the expression for the pressure field in the volume of two fluids for a given flow field;

$$
p_{j}=p_{j 0}+\rho_{j}\left(\dot{\phi}_{j}-\frac{1}{2}\left(\nabla \phi_{j}\right)^{2}-g z\right) .
$$

Now the stress on the interface needs to be included to make the equation system self-contained, by providing 
the required boundary conditions for $\phi_{j}$ on the interface between the two fluids. The pressure jump across the interface is due to the surface tension;

$$
z=\zeta(x, y): \quad p_{1}-p_{2}=-\alpha \nabla \cdot \vec{n} \quad \text { with } \vec{n}=\frac{\nabla F}{|\nabla F|}
$$

where $\alpha$ is the surface tension coefficient and $\vec{n}$ is the unit vector normal to the interface.

The system we consider does not possess any internal instability mechanisms in the absence of vibrations (unlike, e.g., [9, 10]). Vibrations discriminate one of horizontal directions and there are no reasons to expect that close to the threshold of vibration-induced instabilities the excited patterns will experience spatial modulation along the $y$-direction, which is perpendicular to the vibration polarization direction. Furthermore, the linear stability analysis revealed the marginal vibrationinduced instability of the flat-interface state to be longwavelength [3, 6]. Hence, we restrict our consideration to the case of $(x, z)$-geometry and the long-wavelength approximation, $\left|\partial_{x} \vec{v}\right| \ll\left|\partial_{z} \vec{v}\right|$.

\section{GOVERNING EQUATIONS FOR LARGE-SCALE PATTERNS}

\section{A. Derivation of equations}

In this section we derive the governing equation for long-wavelength (or large-scale) patterns. We employ the standard multiscale method with small parameters $\left(T^{-1} / \omega\right)$ and $(h / l)$, where $T$ is the characteristic time scale of the evolution of interface patterns (to be specified below), and $l$ is the reference horizontal length of patterns, $\partial_{x} \sim l^{-1}$. The hierarchy of small parameters and the orders of magnitude of fields will be determined in the course of derivation.

Within the long-wavelength approximation, the solutions to the Laplace equation for $\phi_{j}(x, t)$ satisfying boundary conditions (3) in the most general form read

$$
\begin{array}{r}
\phi_{1}=-a_{1}(t) x+ \\
+\Phi_{1}(x, t)-\frac{1}{2}(h-z)^{2} \Phi_{1 x x}(x, t) \\
+\frac{1}{4 !}(h-z)^{4} \Phi_{1 x x x x}(x, t)-\ldots, \\
\phi_{2}=-a_{2}(t) x+ \\
\Phi_{2}(x, t)-\frac{1}{2}(h+z)^{2} \Phi_{2 x x}(x, t) \\
+\frac{1}{4 !}(h+z)^{4} \Phi_{2 x x x x}(x, t)-\ldots .
\end{array}
$$

Here the ground state (the flat-interface state) is represented by the terms $-a_{j}(t) x ; \Phi_{j}(x, t)$ describe perturbation flow, they are as yet arbitrary functions of $x$ and $t$. After substitution of $p_{j}$ from expression (6) and $\phi_{j}$ from expressions (8)-(9), the condition of stress balance on the interface (7) reads

$$
\begin{array}{r}
p_{1 \infty}-p_{2 \infty}+\rho_{1}\left[-\dot{a}_{1} x+\dot{\Phi}_{1}-\frac{(h-\zeta)^{2}}{2} \dot{\Phi}_{1 x x}-\frac{1}{2}\left(-a_{1}+\Phi_{1 x}-\frac{(h-\zeta)^{2}}{2} \Phi_{1 x x x}\right)^{2}\right. \\
\left.-\frac{\left((h-\zeta) \Phi_{1 x x}\right)^{2}}{2}+\ldots\right] \\
-\rho_{2}\left[-\dot{a}_{2} x+\dot{\Phi}_{2}-\frac{(h+\zeta)^{2}}{2} \dot{\Phi}_{2 x x}-\frac{1}{2}\left(-a_{2}+\Phi_{2 x}-\frac{(h+\zeta)^{2}}{2} \Phi_{2 x x x}\right)^{2}\right. \\
\left.-\frac{\left((h+\zeta) \Phi_{2 x x}\right)^{2}}{2}+\ldots\right] \\
+\left(\rho_{2}-\rho_{1}\right) g \zeta=\alpha \frac{\zeta_{x x}}{\left(1+\zeta_{x}^{2}\right)^{3 / 2}} .
\end{array}
$$

Here "..." stand for terms $\mathcal{O}_{1}\left(\dot{\Phi}_{j} h^{4} / l^{4}\right)+\mathcal{O}_{2}\left(\Phi_{j}^{2} h^{4} / l^{6}\right)+$ $\mathcal{O}_{3}\left(a_{j} \Phi_{j} h^{4} / l^{5}\right)$; here and in what follows, $\mathcal{O}_{j}(Z)$ stand for unspecified contributions of the same order of smallness as their argument $Z$, and index $j$ is used to distinguish several nonidentical contributions to one and the same equation. We specify the order of the neglected terms so 
as to facilitate tracking the correctness of the derivations. The difference of constants $p_{1 \infty}-p_{2 \infty}$ is to be determined from the condition that in the area of vanishing perturbations of the pulsation flow, i.e. $\Phi_{j}(x, t)=$ const, the interface remains flat, i.e. $\zeta(x, t)=0$. This condition yields $p_{1 \infty}-p_{2 \infty}-\left(\rho_{1} a_{1}^{2}(t)-\rho_{2} a_{2}^{2}(t)\right) / 2=0$. One can choose the following units of measurements for length: $L=\sqrt{\alpha /\left[\left(\rho_{2}-\rho_{1}\right) g\right]}$, for time: $T=L / b$, and for the fluid densities: $\rho_{*}$-which mean replacement

$$
\begin{gathered}
(x, z) \rightarrow(L x, L z), \quad t \rightarrow T t, \quad \zeta \rightarrow L \zeta \\
\Phi_{j} \rightarrow\left(L^{2} / T\right) \Phi_{j}, \quad \rho_{i} \rightarrow \rho_{*} \rho_{i}
\end{gathered}
$$

in equations - and rewrite the last equation in the dimensionless form

$$
\begin{aligned}
& B\left[\frac{\rho_{1} a_{1}^{2}-\rho_{2} a_{2}^{2}}{2}+\rho_{1} \dot{\Phi}_{1}-\rho_{2} \dot{\Phi}_{2}-\frac{\rho_{1}(h-\zeta)^{2}}{2} \dot{\Phi}_{1 x x}+\frac{\rho_{2}(h+\zeta)^{2}}{2} \dot{\Phi}_{2 x x}-\frac{\rho_{1}}{2}\left(a_{1}-\Phi_{1 x}+\frac{1}{2}(h-\zeta)^{2} \Phi_{1 x x x}\right)^{2}\right. \\
& \left.\quad+\frac{\rho_{2}}{2}\left(a_{2}-\Phi_{2 x}+\frac{1}{2}(h+\zeta)^{2} \Phi_{2 x x x}\right)^{2}-\frac{\rho_{1}}{2}\left((h-\zeta) \Phi_{1 x x}\right)^{2}+\frac{\rho_{2}}{2}\left((h+\zeta) \Phi_{2 x x}\right)^{2}+\ldots\right]+\zeta=\frac{\zeta_{x x}}{\left(1+\zeta_{x}^{2}\right)^{3 / 2}}
\end{aligned}
$$

Here the dimensionless vibration parameter

$$
B \equiv \frac{\rho_{*} b^{2}}{\sqrt{\alpha\left(\rho_{2}-\rho_{1}\right) g}}=B_{0}+B_{1}
$$

( $\rho_{j}$ is dimensional here), where $B_{0}$ is the critical value of the vibration parameter above which the flat-interface state becomes linearly unstable, $B_{1}$ is a small deviation of the vibration parameter from the critical value. Further, kinematic conditions (4) and (5) turn into

$$
\begin{gathered}
\dot{\zeta}=\left(-(h-\zeta) \Phi_{1 x}+\frac{1}{3 !} h^{3} \Phi_{1 x x x}-a_{1} \zeta+\ldots\right)_{x}, \\
\dot{\zeta}=\left((h+\zeta) \Phi_{2 x}-\frac{1}{3 !} h^{3} \Phi_{2 x x x}-a_{2} \zeta+\ldots\right)_{x} .
\end{gathered}
$$

Here “..." stand for $\mathcal{O}_{1}\left(\Phi_{j} h^{2} \zeta / l^{3}\right)+\mathcal{O}_{2}\left(\Phi_{j} h^{4} / l^{5}\right)$. Equations (11), (13) and (14) form a self-contained equation system.

It is convenient to distinguish two main time-modes in various fields: the average over vibration period part and the pulsation part;

$$
\begin{aligned}
& \zeta=\eta(\tau, x)+\xi(\tau, x) e^{i \omega t}+c . c .+\ldots \\
& \Phi_{j}=\varphi_{j}(\tau, x)+\psi_{j}(\tau, x) e^{i \omega t}+\text { c.c. }+\ldots
\end{aligned}
$$

where $\tau$ is a "slow" time related to the average over vibration period evolution and "..." stand for higher powers of $e^{i \omega t}$.

In order to develop an expansion in small parameter $\omega^{-1}$, we have to adopt a certain hierarchy of smallness of parameters, fields, etc. We adopt small deviation from the instability threshold $B_{1} \sim \omega^{-1}$. Then $\eta \sim \omega^{-1}$ and $\partial_{x} \sim \omega^{-1 / 2}$ (cf. [3, 6]). It is as well established (e.g., [3, 6]) that for finite wavelength perturbations (finite $k \neq 0$ ) $B_{0}(k)=B_{0}(0)+C k^{2}+\mathcal{O}\left(k^{4}\right)$. Generally, the expansion of the exponential growth rate of perturbations in the series for $B_{1}$ near the instability threshold possesses a non-zero linear part, and $B_{0}(k)-B_{0}(0) \sim k^{2}$; therefore, $\partial_{\tau} \sim \mathcal{O}_{1}\left(B_{1}\right)+\mathcal{O}_{2}\left(k^{2}\right) \sim \omega^{-1}$. It is more convenient to determine the order of magnitude of $\xi, \varphi_{j}$ and $\psi_{j}$ in the course of development of the expansion.

Collecting terms with $e^{i \omega t}$ in equations (13) and (14), one finds

$$
\begin{array}{r}
i \omega \xi+\xi_{\tau}=\left(-(h-\eta) \psi_{1 x}+\frac{1}{3 !} h^{3} \psi_{1 x x x}\right. \\
\left.+\xi \varphi_{1 x}-A_{1} \eta+\ldots\right)_{x} \\
i \omega \xi+\xi_{\tau}=\left((h+\eta) \psi_{2 x}-\frac{1}{3 !} h^{3} \psi_{2 x x x}\right. \\
\left.+\xi \varphi_{2 x}-A_{2} \eta+\ldots\right)_{x}
\end{array}
$$

where "..." stand for $\mathcal{O}_{1}\left((\xi \varphi+\eta \psi) h^{2} / l^{4}\right)+\mathcal{O}_{2}\left(\psi h^{4} / l^{6}\right)$. Terms constant with respect to $t$ sum up to

$$
\begin{aligned}
& \eta_{\tau}=\left(-(h-\eta) \varphi_{1 x}+\xi \psi_{1 x}^{*}+c . c .-A_{1} \xi^{*}+c . c .+\ldots\right)_{x}, \\
& \eta_{\tau}=\left((h+\eta) \varphi_{2 x}+\xi \psi_{2 x}^{*}+c . c .-A_{2} \xi^{*}+c . c .+\ldots\right)_{x}
\end{aligned}
$$

where the superscript "*" stands for complex conjugate and "..." stand for $\mathcal{O}_{1}\left((\eta \varphi+\xi \psi) h^{2} / l^{4}\right)+\mathcal{O}_{2}\left(\varphi h^{4} / l^{6}\right)$. The difference of equations (15) and (16) yields $\psi_{j} \sim$ $\omega^{-1 / 2}$, and the difference of (17) and (18) yields $\varphi_{j} \sim$ $\omega^{-1}$. For dealing with non-linear terms in what follows, it is convenient to extract the first correction to $\psi_{j}$ explicitly, i.e. write $\psi_{j}=\psi_{j}^{(0)}+\psi_{j}^{(1)}+\ldots$, where $\psi_{j}^{(1)} \sim \omega^{-1} \psi_{j}^{(0)} \sim \omega^{-3 / 2}$. Equation (15) (or (16)) yields in the leading order $\left(\sim \omega^{-3 / 2}\right)$

$$
\xi=\frac{i}{\omega}\left(h \psi_{1 x}+A_{1} \eta\right)_{x} \sim \omega^{-\frac{5}{2}} .
$$


Considering the difference of (16) and (15), one has to keep in mind that we are interested in localized patterns for which $\Phi_{j x}(x= \pm \infty)=0, \zeta(x= \pm \infty)=0$. Hence, this difference can be integrated with respect to $x$, taking the form

$$
\begin{aligned}
h\left(\psi_{1}+\psi_{2}\right)_{x} & -\eta\left(\psi_{1}-\psi_{2}\right)_{x}-\frac{1}{6} h^{3}\left(\psi_{1}+\psi_{2}\right)_{x x x} \\
& -\xi\left(\varphi_{1}-\varphi_{2}\right)_{x}+\left(A_{1}-A_{2}\right) \eta+\cdots=0,
\end{aligned}
$$

which yields in the first two orders of smallness

$$
\begin{gathered}
h\left(\psi_{1}^{(0)}+\psi_{2}^{(0)}\right)_{x}=-\left(A_{1}-A_{2}\right) \eta=-\frac{\rho_{2}-\rho_{1}}{\rho_{2}+\rho_{1}} \eta, \\
h\left(\psi_{1}^{(1)}+\psi_{2}^{(1)}\right)_{x}=\left(\psi_{1}^{(0)}-\psi_{2}^{(0)}\right)_{x} \eta+\frac{1}{6} h^{3}\left(\psi_{1}^{(0)}+\psi_{2}^{(0)}\right)_{x x x} .
\end{gathered}
$$

The difference and the sum of equations (17) and (18) yield in the leading order, respectively,

$$
\begin{gathered}
\varphi_{1}=-\varphi_{2} \equiv \varphi, \\
\eta_{\tau}=-h \varphi_{x x} .
\end{gathered}
$$

Let us now consider equation (11). We will collect groups of terms with respect to power of $e^{i \omega t}$ and the order of smallness in $\omega^{-1}$.

$\sim \omega^{+\frac{1}{2}} e^{i \omega t}$ :

$$
i \omega B_{0}\left(\rho_{1} \psi_{1}^{(0)}-\rho_{2} \psi_{2}^{(0)}\right)=0 .
$$

We introduce

$$
\psi^{(0)} \equiv \rho_{j} \psi_{j}^{(0)} .
$$

The last equation and equation (20) yield

$$
\psi_{x}^{(0)}=-\frac{1}{h} \frac{\rho_{1} \rho_{2}\left(\rho_{2}-\rho_{1}\right)}{\left(\rho_{2}+\rho_{1}\right)^{2}} \eta .
$$

$\simeq \omega^{0} e^{i \omega t}$

No contributions.

$\simeq \omega^{-\frac{1}{2}} e^{i \omega t}$

$$
\begin{gathered}
i \omega B_{1} \underbrace{\left(\rho_{1} \psi_{1}^{(0)}-\rho_{2} \psi_{2}^{(0)}\right)}_{=0}+i \omega B_{0}\left(\rho_{1} \psi_{1}^{(1)}-\rho_{2} \psi_{2}^{(1)}\right) \\
+B_{0} \underbrace{\left(\rho_{1} \psi_{1}^{(0)}-\rho_{2} \psi_{2}^{(0)}\right)_{\tau}}_{=0} \\
+i \omega B_{0} \frac{h^{2}}{2} \underbrace{\left(\rho_{2} \psi_{2 x x}^{(0)}-\rho_{1} \psi_{1 x x}^{(0)}\right.}_{=0})=0 .
\end{gathered}
$$

(We marked the combinations which are known to be zero from the leading order of expansion.) Similarly to (24), we introduce

$$
\psi^{(1)} \equiv \rho_{j} \psi_{j}^{(1)} .
$$

The last equation and equation (21) yield

$$
\begin{aligned}
& \begin{aligned}
\psi_{x}^{(1)} & =\frac{1}{h} \frac{\rho_{2}-\rho_{1}}{\rho_{2}+\rho_{1}} \psi_{x}^{(0)} \eta+\frac{h^{2}}{6} \psi_{x x x}^{(0)} \\
& =-\frac{\rho_{1} \rho_{2}\left(\rho_{2}-\rho_{1}\right)^{2}}{h^{2}\left(\rho_{2}+\rho_{1}\right)^{3}} \eta-\frac{h \rho_{1} \rho_{2}\left(\rho_{2}-\rho_{1}\right)}{6\left(\rho_{2}+\rho_{1}\right)^{2}} \eta_{x x} . \\
& \sim \omega^{-1}\left(e^{i \omega t}\right)^{0}:
\end{aligned}
\end{aligned}
$$

$$
B_{0}\left[-\rho_{2}\left(A_{2} \psi_{2 x}^{(0) *}+\text { c.c. }\right)+\rho_{1}\left(A_{1} \psi_{1 x}^{(0) *}+\text { c.c. }\right)\right]+\eta=0 .
$$

Substituting (24) and (25) into the last equation gives

$$
\left[-\frac{2 B_{0} \rho_{1} \rho_{2}\left(\rho_{2}-\rho_{1}\right)^{2}}{h\left(\rho_{2}+\rho_{1}\right)^{3}}+1\right] \eta=0 .
$$

Thus we obtain the solvability condition, which poses a restriction on $B_{0}$; this restriction determines the linear instability threshold

$$
B_{0}=\frac{\left(\rho_{2}+\rho_{1}\right)^{3} h}{2 \rho_{1} \rho_{2}\left(\rho_{2}-\rho_{1}\right)^{2}} .
$$

$\underline{\sim \omega^{-2}\left(e^{i \omega t}\right)^{0}}$ : (using (22) for $\left.\varphi_{j}\right)$

$$
\begin{aligned}
& B_{1} \underbrace{\left[-\rho_{2}\left(A_{2} \psi_{2 x}^{(0) *}+c . c .\right)+\rho_{1}\left(A_{1} \psi_{1 x}^{(0) *}+\text { c.c. }\right)\right]}_{=-\eta / B_{0}} \\
& \quad+B_{0}\left[\left(\rho_{2}+\rho_{1}\right) \varphi_{\tau}+\rho_{2}\left|\psi_{2 x}^{(0)}\right|^{2}\right. \\
& -\rho_{2}\left(A_{2} \psi_{2 x}^{(1) *}+\text { c.c. }-A_{2} \frac{h^{2}}{2} \psi_{2 x x x}^{(0) *}+\text { c.c. }\right)-\rho_{1}\left|\psi_{1 x}^{(0)}\right|^{2} \\
& \left.\quad+\rho_{1}\left(A_{1} \psi_{1 x}^{(1) *}+\text { c.c. }-A_{1} \frac{h^{2}}{2} \psi_{1 x x x}^{(0) *}+\text { c.c. }\right)\right]=\eta_{x x} .
\end{aligned}
$$

Substituting $\psi_{j}^{(n)}$ from (24)-(27), one can rewrite the latter equation as

$$
\begin{array}{r}
-\frac{B_{1}}{B_{0}} \eta+B_{0}\left[\left(\rho_{2}+\rho_{1}\right) \varphi_{\tau}-\frac{\rho_{2}-\rho_{1}}{\rho_{2} \rho_{1}}\left(\frac{\rho_{1} \rho_{2}\left(\rho_{2}-\rho_{1}\right) \eta}{h\left(\rho_{2}+\rho_{1}\right)^{2}}\right)^{2}\right. \\
-\frac{2 \rho_{1} \rho_{2}\left(\rho_{2}-\rho_{1}\right)^{3}}{h^{2}\left(\rho_{2}+\rho_{1}\right)^{4}} \eta^{2}-\frac{h \rho_{1} \rho_{2}\left(\rho_{2}-\rho_{1}\right)^{2}}{3\left(\rho_{2}+\rho_{1}\right)^{3}} \eta_{x x} \\
\left.+h^{2} \frac{\rho_{1} \rho_{2}\left(\rho_{2}-\rho_{1}\right)^{2}}{h\left(\rho_{2}+\rho_{1}\right)^{3}} \eta_{x x}\right]=\eta_{x x} .
\end{array}
$$

Together with equation (23) the latter equation form the final system of governing equations for long-wavelength perturbations of the flat-interface state:

$$
\left\{\begin{aligned}
B_{0}\left(\tilde{\rho}_{2}+\tilde{\rho}_{1}\right) \tilde{\varphi}_{\tilde{\tau}} & =\left[1-\frac{\tilde{h}^{2}}{3}\right] \tilde{\eta}_{\tilde{x} \tilde{x}}+\frac{3}{2 \tilde{h}} \frac{\tilde{\rho}_{2}-\tilde{\rho}_{1}}{\tilde{\rho}_{2}+\tilde{\rho}_{1}} \tilde{\eta}^{2}+\frac{B_{1}}{B_{0}} \tilde{\eta}, \\
\tilde{\eta}_{\tilde{\tau}} & =-\tilde{h} \tilde{\varphi}_{\tilde{x} \tilde{x}}
\end{aligned}\right.
$$


Here we explicitly mark the dimensionless variables and parameters with the tilde sign to distinguish them from original dimensional variables and parameters. Above in this paragraph, the tilde sign was omitted to make calculations possibly less laborious. For convenience we explicitly specify how to read rescaling (10) with the tildenotation: $x=L \tilde{x}, t=(L / b) \tilde{t}, \rho_{i}=\rho_{*} \tilde{\rho}_{i}$, etc. The expression for $B_{0}$ (28) in the original dimensional terms reads

$$
B_{0}=\frac{\rho_{*}\left(\rho_{2}+\rho_{1}\right)^{3} h}{2 \rho_{1} \rho_{2}\left(\rho_{2}-\rho_{1}\right)^{2}} \sqrt{\frac{\left(\rho_{2}-\rho_{1}\right) g}{\alpha}} .
$$

We remark that equation system (29) is valid for $B_{1}$ small compared to $B_{0}$, otherwise one cannot stay within the long-wavelength approximation. On rare occasions it is possible to use long-wavelength for finite deviations from the linear instability threshold and derive certain information on the system dynamics (e.g., in [11] for Soret-driven convection from localized sources of heat or solute in a thin porous layer, an unavoidable appearance of patterns similar to hydraulic jumps [12] was predicted within the long-wavelength approximation though for a finite deviation from the linear instability threshold).

\section{B. On the long-wavelength character of the linear instability}

In the text above, we relied on the fact that instability is long-wavelength for thin enough layers. Now we have appropriate quantifiers to specify quantitatively, what "thin enough" means. According to [3], we require that $\tilde{h}<\sqrt{3}$. Remarkably, we can see a footprint of this fact from equation system (29) with multiplier $\left[1-\tilde{h}^{2} / 3\right]$ ahead of $\tilde{\eta}_{\tilde{x} \tilde{x}}$. Indeed, the exponential growth rate $\tilde{\lambda}$ of linear normal perturbations $(\tilde{\eta}, \tilde{\varphi}) \propto \exp (\tilde{\lambda} \tilde{t}+i \tilde{k} \tilde{x})$ of the trivial state obeys

$$
\tilde{\lambda}^{2}=\frac{\tilde{h} \tilde{k}^{2}}{B_{0}\left(\tilde{\rho}_{2}+\tilde{\rho}_{1}\right)}\left(-\left[1-\frac{\tilde{h}^{2}}{3}\right] \tilde{k}^{2}+\frac{B_{1}}{B_{0}}\right) .
$$

Below the linear instability threshold of infinitely long wavelength perturbations, i.e. for $B_{1}<0$, there are no growing perturbations for $\tilde{h}<\sqrt{3}$, while the perturbations with large enough $\tilde{k}$ grow for $\tilde{h}>\sqrt{3}$. Of course, this analysis of equation system (29) only highlights the long-wavelength character of the linear instability, since it deals with the limit of small $\tilde{k}$ and does not provide information on the linear stability for finite $\tilde{k}$. A comprehensive proof of the long-wavelength character of the instability for $\tilde{h}<\sqrt{3}$ comes from [3].

In the following we will consider system behavior below the linear instability threshold, i.e. for negative $B_{1}$. It is convenient to make further rescaling of coordinates and variables:

$$
\begin{aligned}
& \tilde{x} \rightarrow x \sqrt{\frac{B_{0}}{\left(-B_{1}\right)}\left[1-\frac{\tilde{h}^{2}}{3}\right]}, \\
& \tilde{t} \rightarrow t \sqrt{\frac{\tilde{\rho}_{2}-\tilde{\rho}_{1}}{\tilde{h}} \frac{B_{0}^{3}}{B_{1}^{2}}\left[1-\frac{\tilde{h}^{2}}{3}\right]}, \\
& \tilde{\eta} \rightarrow \eta \tilde{h} \frac{\tilde{\rho}_{2}+\tilde{\rho}_{1}}{\tilde{\rho}_{2}-\tilde{\rho}_{1}} \frac{\left(-B_{1}\right)}{B_{0}}, \\
& \tilde{\varphi} \rightarrow \varphi \sqrt{\frac{\left(\tilde{\rho}_{2}+\tilde{\rho}_{1}\right)^{2}}{\left(\tilde{\rho}_{2}-\tilde{\rho}_{1}\right)^{3}} \frac{B_{1}^{2}}{\tilde{h} B_{0}^{3}}\left[1-\frac{\tilde{h}^{2}}{3}\right]} .
\end{aligned}
$$

We note that this implies the following rescaling of initial dimensional coordinates and variables:

$$
\begin{aligned}
& x \rightarrow x L \sqrt{\frac{B_{0}}{\left(-B_{1}\right)}\left[1-\frac{h^{2}}{3 L^{2}}\right]}, \\
& t \rightarrow t \sqrt{\frac{\rho_{2}-\rho_{1}}{\rho_{*}} \frac{L^{3} B_{0}^{3}}{h b^{2} B_{1}^{2}}\left[1-\frac{h^{2}}{3 L^{2}}\right]}, \\
& \eta \rightarrow \eta h \frac{\rho_{2}+\rho_{1}}{\rho_{2}-\rho_{1}} \frac{\left(-B_{1}\right)}{B_{0}}, \\
& \varphi \rightarrow \varphi \sqrt{\frac{\rho_{*}\left(\rho_{2}+\rho_{1}\right)^{2}}{\left(\rho_{2}-\rho_{1}\right)^{3}} \frac{L^{3} B_{1}^{2}}{h b^{2} B_{0}^{3}}\left[1-\frac{h^{2}}{3 L^{2}}\right]} .
\end{aligned}
$$

After this rescaling, equation system (29) takes the zeroparametric form;

$$
\begin{aligned}
\dot{\varphi} & =\eta_{x x}+\frac{3}{2} \eta^{2}-\eta, \\
\dot{\eta} & =-\varphi_{x x} .
\end{aligned}
$$

The derivation of the latter equation system itself is one of the main results we report with this paper, as it allows consideration of the evolution of quasi-steady patterns in the two-layer fluid system under the action of the vibration field.

\section{The 'plus' Boussinesq equation and the original Boussinesq equation for gravity waves in shallow water}

The equation system (34)-(35) can be rewritten in the form of a 'plus' Boussinesq equation (plus BE);

$$
\ddot{\eta}-\eta_{x x}+\left(\frac{3}{2} \eta^{2}+\eta_{x x}\right)_{x x}=0 .
$$


Meanwhile, the original Boussinesq equation B (BE B) for gravity waves in a shallow water layer [13] or in a two-layer system without vibrations [14] reads

$$
\ddot{\eta}-\eta_{x x}-\left(\frac{3}{2} \eta^{2}+\eta_{x x}\right)_{x x}=0 .
$$

Both systems are fully integrable and multi-soliton solutions are known for them from the literature (e.g., $15-$ 17]). However, their dynamics is essentially different; the original BE B suffers from the short-wave instability, while the plus BE is free from this instability. Solitons in the plus BE can be unstable, decaying into pairs of stable solitons or experiencing explosive formation of sharp peaks in finite time [17-19]. In the sections below we will provide overview of the soliton dynamics for equation (36) in relation to the fluid dynamical system we deal with. Prior to doing so, in this subsection, we would like to focus more on discussion of different kinds of the generalized Boussinesq equation and their relationships with dynamics of systems of fluid layers.

Small-amplitude gravity waves in shallow water are governed by the set A Boussinesq equations (equation system (25) in [13]) which read in our terms after proper rescaling as

$$
\left\{\begin{aligned}
\dot{\eta}+\varphi_{x x} & =-\left(\eta \varphi_{x}\right)_{x}+\frac{1}{6} \varphi_{x x x x}, \\
\dot{\varphi}+\eta & =-\frac{1}{2}\left(\varphi_{x}\right)^{2}+\frac{1}{2} \dot{\varphi}_{x x},
\end{aligned}\right.
$$

where the terms in the right hand side of equations are small, i.e., both nonlinearity and dispersion are small. To the leading corrections pertaining to nonlinearity and dispersion, the latter equation system can be recast as

$$
\ddot{\eta}-\eta_{x x}=\left(\left(\varphi_{x}\right)^{2}+\frac{1}{2} \eta^{2}+\eta_{x x}\right)_{x x},
$$

where small terms are collected in the r.h.s. part of the equation. For waves propagating in one direction $\partial_{x} \approx$ $\pm \partial_{t}$ and, to the leading corrections, one can make substitution $\left(\varphi_{x}\right)^{2} \approx(\dot{\varphi})^{2} \approx \eta$, which yields equation (37). Thus, the Boussinesq equation for the classical problem of waves in shallow water is not only inaccurate far from the edge of the spectrum of soliton speed (near $c=1$ ) but is also inappropriate for consideration of collisions of counterpropagating waves (as $\left|\varphi_{x}\right| \neq|\dot{\varphi}|$ for them). In contrast, the equations we derived for our physical system are accurate close to the vibration-induced instability threshold for the entire range of soliton speeds and all kinds of soliton interactions as long as the profile remains smooth.

It is also noteworthy, that character of the original Boussinesq equation $\mathrm{B}$ is inherent to the dynamics of inviscid fluid layers in force fields and does not change without special external fields, the action of which cannot be formally represented by any correction to the gravity. The case of a vibration field turns out to be one such and yields the dynamics governed by the plus BE.

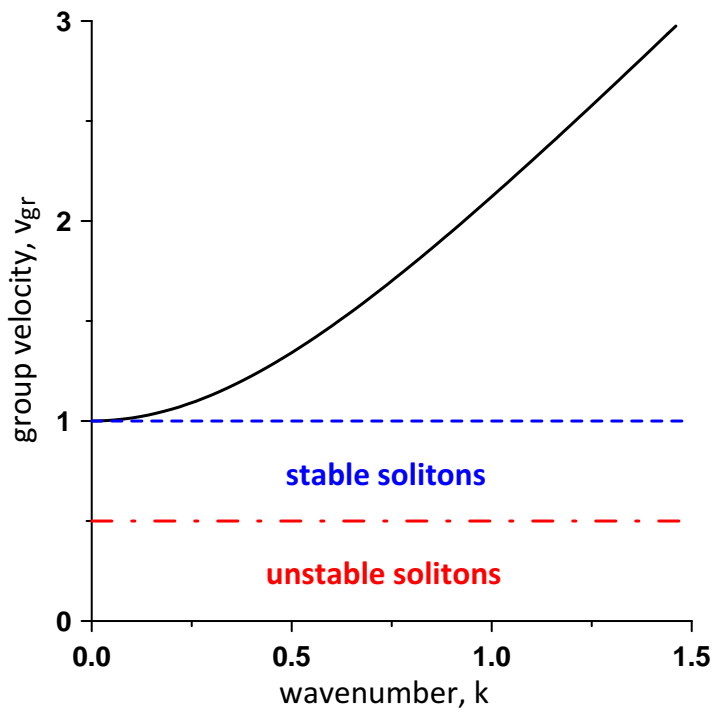

FIG. 2: Group velocity $v_{\text {gr }}$ of linear waves (black solid line) vs the wavenumber $k$. The group velocity for all wave packages is larger than the maximal soliton propagation velocity $c=1$. (Soliton stability is discussed in the text below.)

\section{LONG WAVES BELOW THE LINEAR INSTABILITY THRESHOLD}

In this section we consider waves in the dynamic system (34)-(35). In equations (33), one can see how the rescaling of each coordinate and variable depends on $\left(-B_{1}\right)=B_{0}-B$. From these dependencies it can be seen, that for patterns in the dynamic system (34)-(35) the corresponding patterns in real time-space will obey the following scaling behavior near the linear instability threshold: spatial extent $x_{*} \propto 1 / \sqrt{B_{0}-B}$, reference time $t_{*} \propto 1 /\left(B_{0}-B\right)$, reference profile deviation $\eta_{*} \propto\left(B_{0}-B\right)$.

\section{A. Linear waves: dispersion equation, group velocity}

Let us first describe propagation of small perturbation, linear waves, in the dynamic system (34)-(35). For normal perturbations $(\eta, \varphi) \propto \exp (-i \Omega t+i k x)$ the oscillation frequency reads

$$
\Omega(k)=k \sqrt{1+k^{2}} .
$$

The corresponding phase velocity is

$$
v_{\mathrm{ph}}=\Omega / k=\sqrt{1+k^{2}},
$$

and the group velocity, which describes propagation of envelopes of wave packages, is

$$
v_{\mathrm{gr}}=\frac{\mathrm{d} \Omega}{\mathrm{d} k}=\frac{1+2 k^{2}}{\sqrt{1+k^{2}}} .
$$


One can see that the minimal group velocity is 1 and the group velocity $v_{\mathrm{gr}}$ monotonously increases as wavelength decreases (see Fig.21).

\section{B. Solitons}

The dynamic system (34)-35) admits timeindependent-profile solutions, solitons $\eta(x, t)=\eta(x-c t)$, where $c$ is the soliton velocity. With identical equality $\partial_{t} \eta(x-c t)=-c \partial_{x} \eta(x-c t)$, for localized patterns, which vanish at $x \rightarrow \pm \infty$, equation (35) can be once integrated and yields $\varphi^{\prime}=c \eta$ (here the prime denotes the differentiation with respect to argument). Eq. (34) takes the form

$$
0=\eta^{\prime \prime}+\frac{3}{2} \eta^{2}-\left(1-c^{2}\right) \eta .
$$

The latter equation admits the soliton solution

$$
\eta_{0}(x, t)=\frac{1-c^{2}}{\cosh ^{2} \frac{\sqrt{1-c^{2}}(x \pm c t)}{2}},
$$

the propagation direction $(+c$ or $-c)$ is determined by the flow, $\varphi^{\prime}= \pm c \eta$. The family of soliton solutions turns out to be one-parametric, parameterized by the speed $c$ only. The speed $c$ varies within the range $[0,1]$; standing soliton $(c=0)$ is the sharpest and the tallest one and for the fastest solitons, $c \rightarrow 1$, the spatial extent tends to infinity, while the height tends to 0 .

Considering in the same way a non-rescaled equation system (29), one can see, that for a given physical system with vibration parameter $B$ as a control parameter, the shape of a soliton solution is controlled by combination

$$
\left[\left(-B_{1}\right) / B_{0}-\tilde{c}^{2} \tilde{h}^{-1} B_{0}\left(\tilde{\rho}_{2}+\tilde{\rho}_{1}\right)\right] .
$$

This means that one and the same interface inflection soliton can exist for different values of $B$, though, since the shape-controlling parameter (45) should be the same, the non-rescaled soliton run speed $\tilde{c}$ grows as the departure from the threshold $\left(-B_{1}\right)$ increases.

Since $v_{\mathrm{gr}} \geq 1$ (see Fig. (2) and $c^{2} \leq 1$, solitons of arbitrary height travel slower than any small perturbations of the flat-interface state. The maximal speed of solitons, $c_{\max }=1$, coincides with the minimal group velocity of linear waves. This yields notable information on the system dynamics. Fast solitons with $c$ tending to 1 from below are extended and have a small height (see equation (44)), while envelopes of long linear waves propagate with velocity $v_{\mathrm{gr}}$ tending to 1 from above. This means that envelopes of small-height soliton packages travel faster than solitons in these packages. The issue of the generality of situations where the ranges of the possible soliton velocities and the group velocities of linear waves do not overlap but only touch each other is interestingly addressed in 20, 21] from the view point of emission of wave packages by the soliton (or the impossibility of such an emission).

\section{Stability of solitons}

The stability properties of solitons in the 'plus' Boussinesq equation were addressed in literature [16, 18, 19]; in [18] the solitons with $1 / 2 \leq c \leq 1$ were proved to be stable and in [19] the solitons with $c<1 / 2$ were proved to be unstable. One can add more subtle details to this information: the spectrum of Lyapunov exponents (exponential growth rate) and the dependence of the scenario of nonlinear growth of perturbations on the initial perturbation.

The problem of linear stability of the soliton $\eta_{0}(x-c t)$ to perturbations $\left(e^{\lambda t} \eta_{1}\left(x_{1}\right), e^{\lambda t} \varphi_{1}\left(x_{1}\right)\right)$ in the copropagating reference frame $x_{1}=x-c t$ reads

$$
\begin{aligned}
\lambda \varphi_{1}+c \varphi_{1}^{\prime} & =\eta_{1}^{\prime \prime}+3 \eta_{0}\left(x_{1}\right) \eta_{1}-\eta_{1}, \\
\lambda \eta_{1}+c \eta_{1}^{\prime} & =-\varphi_{1}^{\prime \prime}
\end{aligned}
$$

with boundary conditions

$$
\eta_{1}( \pm \infty)=\varphi_{1}( \pm \infty)=0 .
$$

The eigenvalue problem (46)-(48) was solved numerically with employment of the shooting method. The spectra of eigenvalues $\lambda$ for different $c$ are plotted in Fig. 3 and the first two eigenmodes of perturbations of the standing soliton $(c=0)$ are plotted in Fig.4(b). In Fig. 4 (a), one can see the exponential growth rate $\operatorname{Re}(\lambda)$ of perturbations; the standing and slow solitons with $c<0.5$ are unstable, while the fast solitons with $c \geq 0.5$ are stable.

The scenarios of evolution of unstable solitons were observed numerically by means of direct numerical simulation of the dynamic system (34)-(35) with the finite difference method in an $x$-domain of length 200 with periodic boundary conditions and the space step size $h_{x}=0.05$. 26] As in [15 17], two possible scenarios were observed: (i) soliton explosion with formation of a finite amplitude relief or, possibly, layer rupture; (ii) fallingapart of the soliton into two stable solitons (Fig. (5) . Since the phase space of the system is infinite-dimensional, the problem of discrimination of the initial perturbations leading to explosion and those leading to falling-apart may be generally nontrivial. However, in Fig. 3 one can see that there is only one instability mode for $c<1 / 2$ and the nonlinear evolution of perturbations turns out to depend only on projection of the small initial perturbation on this unstable direction. If the instability mode is normalized in such a way that $\eta_{1}\left(x_{1}=0\right)>0$ (cf. Fig.4(b)), the initial perturbations with a positive scalar product with the instability mode lead to explosion, while the perturbations with a negative scalar product lead to falling-apart into two stable solitons.

\section{Soliton gas}

In Fig. 6, a sample of the system dynamics from arbitrary initial conditions is presented in domain $x \in[0 ; 250]$ 

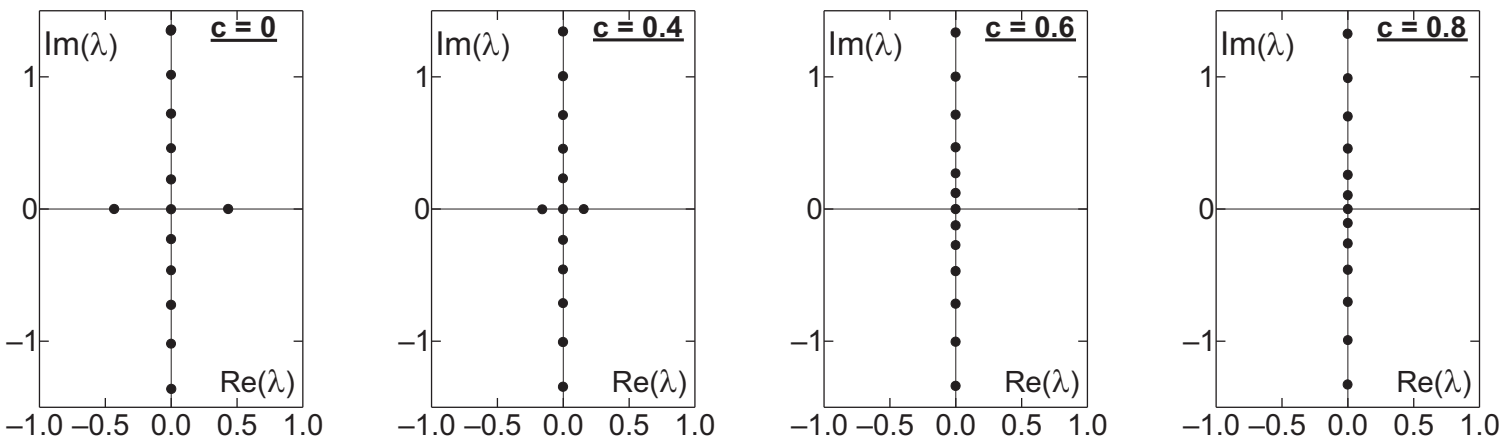

FIG. 3: Spectra of eigenvalues $\lambda$ of the problem (46)-48) of linear stability of solitons with speed $c$ specified in plots. The real part of $\lambda$ is the exponential growth rate of perturbations; for $c=0$ and $c=0.4$, one can see existence of one mode with positive $\operatorname{Re}(\lambda)$, i.e. instability mode, while for $c=0.6$ and $c=0.8$, all the perturbations oscillate without growth. In Fig. 4 (b), the instability mode and the Goldstone mode of neutral stability (invariance to the shifts of soliton in space) are plotted for standing solitons $(c=0)$.

with periodic boundary conditions. One can see that, beyond the locations of formation of singularities [22], this dynamics can be well treated as the kinetics of a gas of stable solitons. For wave dynamics in soliton-bearing systems, statistical physics approaches which describe the dynamics of dense soliton gases can be developed 23.

Knowing that the system dynamics can be viewed as a kinetics of a soliton gas, we can readdress the question of relationships between the group velocity of linear waves and the speed of solitons. The fast solitons with $c \rightarrow 1$ have height $\eta_{\max }=\left[1-c^{2}\right] \rightarrow 0$ and width $\delta \propto 1 / \sqrt{1-c^{2}} \rightarrow \infty$, i.e. must obey the laws established for the linear waves with wavenumber $k \rightarrow 0$. Meanwhile for the latter waves we know the group velocity $v_{\mathrm{gr}}=1+(3 / 2) k^{2}+\mathcal{O}\left(k^{4}\right)$ [see Eq. (42)]. Thus, the envelopes of traveling solitons always travel faster than these solitons. For an envelope of a nearly monochromatic wave the possibility of such a behavior is obvious, while for a gas of quasi-particles additional explanations are needed. For waves of density of quasi-particles (correspond to waves of envelope) it is actually possible to travel faster than the fastest particles if these particles have a finite "collision diameter". (For a better intuition on this, one can imagine the elastic collision of two hard spheres moving along a line. These spheres exchange their momentums from the distance equal to the sum of their radii. If they are identified only by the momentum, they effectively jump for the distance of their interaction and proceed to move with the initial momentums.) Indeed, colliding copropagating solitons exchange their momentums, which means they efficiently exchange their locations, not crossing one another, but approaching for a certain finite distance, the collision diameter. Thus the momentum efficiently jumps in the direction of soliton motion for this distance and, during one and the same time interval, the wave in a gas can cover a longer distance than the gas quasi-particles.

As soon as one can speak of the soliton density waves in a soliton gas, the question of the criterion for this gas to be considered as a continuous medium or a vacuum arises. For instance, it is obvious that one cannot speak of density waves or envelope waves for a system state with a single soliton; this is a vacuum. Whereas, for a continuous medium the concept of the group velocity should work well. Let us consider a gas of solitons with characteristic width $\delta \gg 1$, i.e., quasi-particle speed $c^{2}=$ $1-\delta^{-2}$ [see Eq. (44)]. The signal transfer speed due to collisions (as described in the paragraph above) is larger that the particle speed, according to $v_{\mathrm{gr}} \approx c /(1-n \delta)$, where $n$ is the soliton number density. Mathematically, the criterion for $n$ is of interest. For us to be able to consider the soliton gas as a continuous medium, with the group velocity featured by Eq. (42), $v_{\mathrm{gr}}$ should at least reach 1 . Hence, $c /\left(1-n_{\min } \delta\right)=1$, which can be rewritten as $1-\delta^{-2} / 2=1-n_{\min } \delta$, and one finally finds

$$
n_{\min } \sim \delta^{-3} .
$$

Interestingly, the last equation means that the maximal characteristic inter-soliton distance $\delta_{*}=1 / n_{\min }$ for the gas to be a continuous medium but not a vacuum scales with the soliton width $\delta$ as

$$
\delta_{*} \sim \delta^{3} .
$$

\section{CONCLUSION}

We have considered the dynamics of patterns on the internal surface of the horizontal two-layer system of inviscid fluids subject to tangential vibrations. For thin layers $\left(h<\sqrt{3 \alpha /\left[\left(\rho_{2}-\rho_{1}\right) g\right]}\right)$ the instability is known to be long-wavelength and subcritical [3, [6]. The governing equations for long-wavelength patterns below the linear instability threshold have been derived - equation system (34) - (35) - allowing for the first time theoretical analysis for time-dependent patterns in the system and for stability of time-independent (quasi-steady) patterns. We note that the stability analysis for the only time-independent localized patterns in the system, standing solitons, has revealed them to be unstable. 

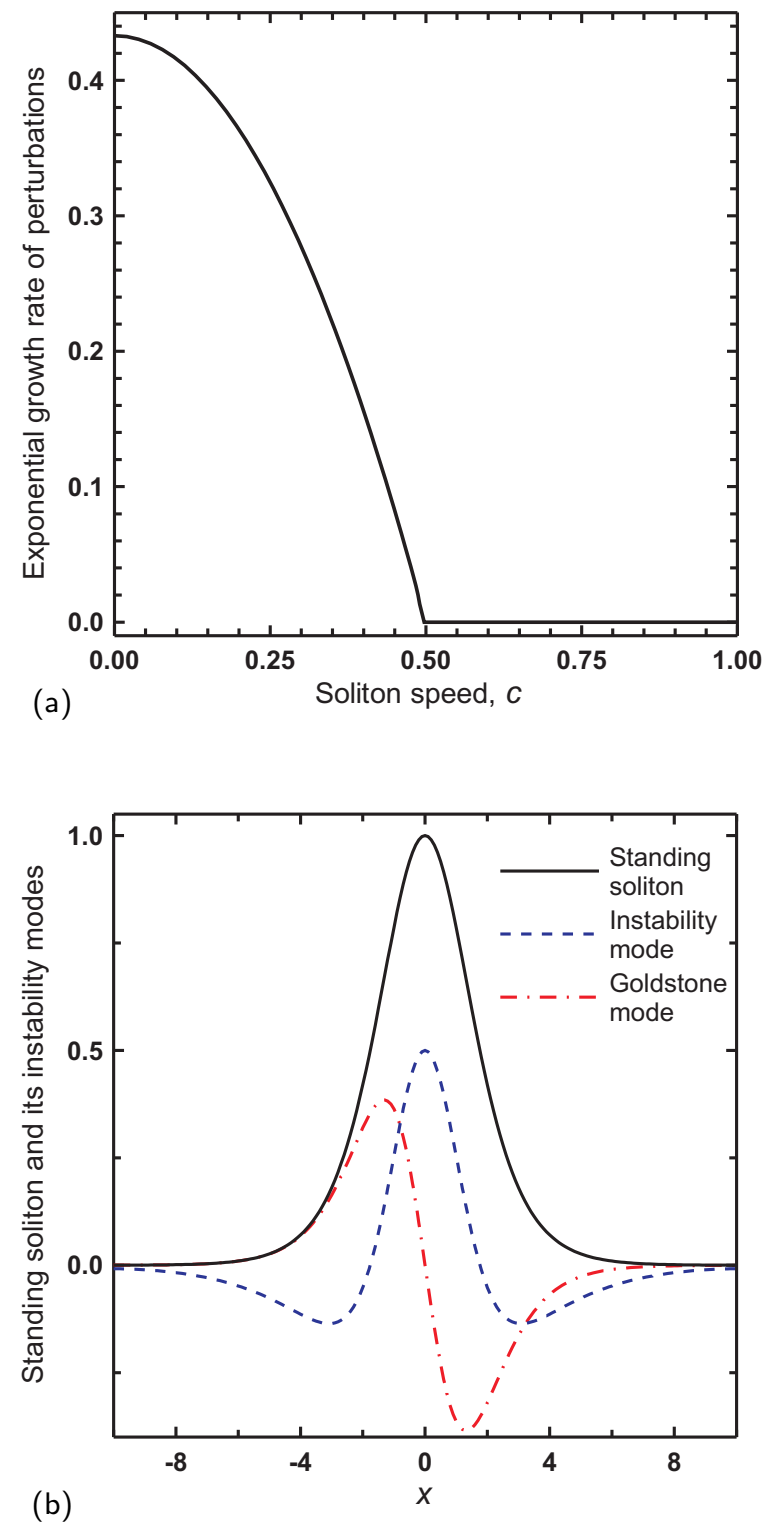

FIG. 4: (a): Exponential growth rate $\operatorname{Re}(\lambda)$ of perturbations of soliton as a function of the soliton speed $c$. (b): The instability mode and the Goldstone mode of neutral stability (invariance to the shifts of soliton in space) of the standing soliton $(c=0)$.

The system dynamics is found to be governed by dynamic system (34)-(35) which is equivalent to the 'plus' Boussinesq equation. For dynamic system (34)(35), one-parametric family of localized solutions of time-independent profile, solitons, exists (equation (44)). These solitons are up-standing embossments of the interface (cf. black curve in Fig. 4(b)) and are parameterized by the soliton speed $c$ only, which varies from $c=0$ (the tallest and sharpest solitons) to $c=1$ (solitons with width tending to infinity and height tending to zero). The standing and slow solitons $(c<1 / 2)$ are unstable 19], while the fast solitons $(c \geq 1 / 2)$ are stable [18]. The group velocity of linear waves in the sys-
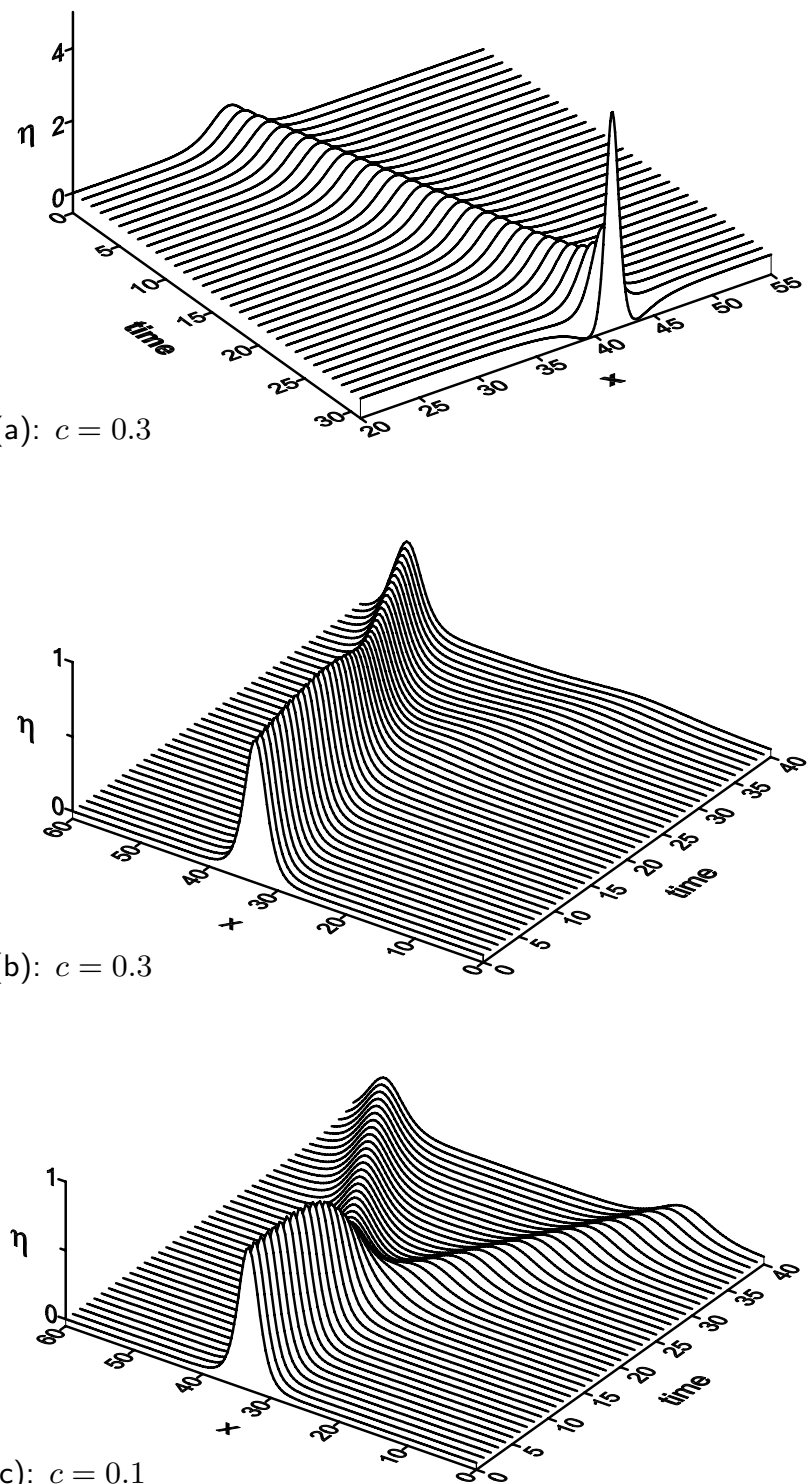

FIG. 5: (a): The unstable soliton (44) of $c=0.3$ with tiny positive perturbation explodes leading to the formation of a finite amplitude relief, possibly layer rupture. (b): The same unstable soliton $(c=0.3)$ with tiny negative perturbation falls apart into two fast stable solitons. (c): Falling-apart of the unstable soliton with $c=0.1$.

tem is $v_{\mathrm{gr}} \geq 1$, meaning that all the solitons travel more slowly than any wave packages of small perturbations of the flat-interface state.

Two scenarios of development of the instability of slow solitons are possible, depending on the initial perturbation: explosion (probably leading to further layer rupture) and splitting into a pair of fast stable solitons. No other localized waves have been detected with direct numerical simulation, meaning that this one-parameter family of solitons is the only localized waves in the system. The system dynamics can be fully represented as the kinetics of gas of solitons before an explosion (and after it). 


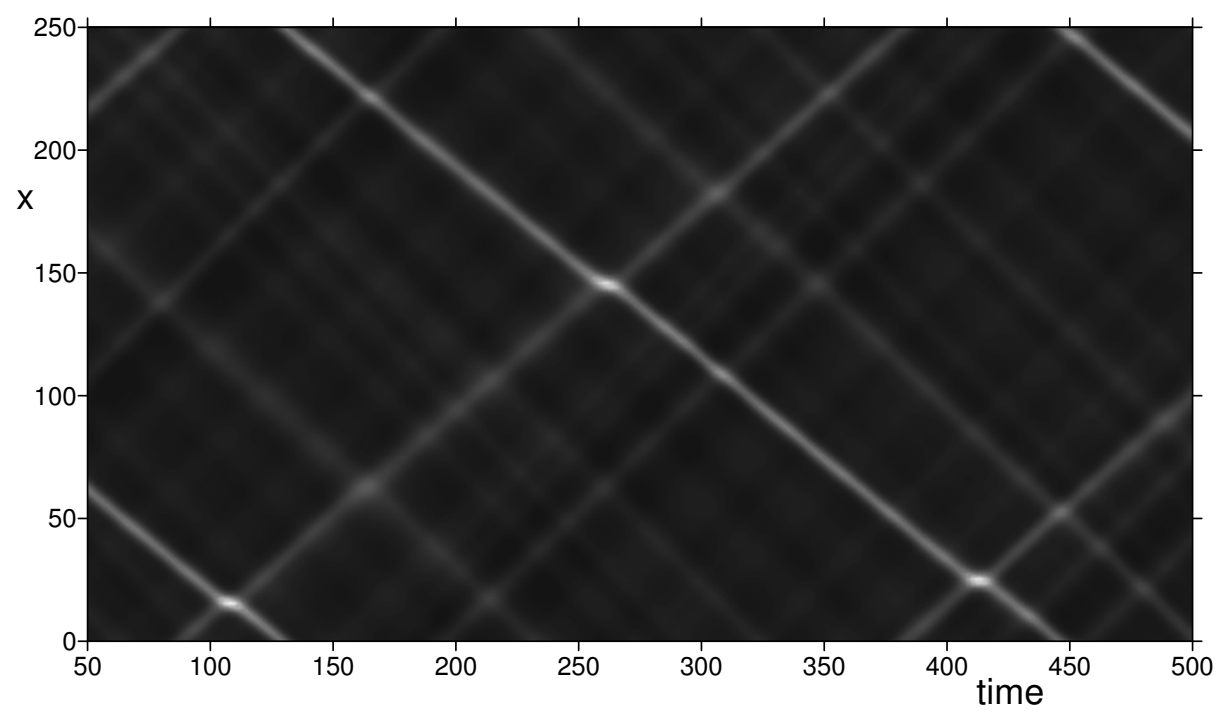

FIG. 6: Sample evolution of the dynamic system (34)-35ith arbitrary smooth initial conditions. The dynamics can be viewed as a kinetics of a gas of stable (fast) solitons.

It is not possible to compare our results to the results presented by Wolf 1, 2] in detail. Wolf presented the wave patterns of the interface for the inverted state (the heavy liquid above the light one) above the linear instability threshold of the flat-interface non-inverted state. Meanwhile, we consider waves on the interface for the non-inverted state below the threshold, and our nontrivial findings pertain specifically to this case but not to the case of the inverted stratification.
We are thankful to Dr. Maxim V. Pavlov and Dr. Takayuki Tsuchida for their useful comments on the work and drawing our attention to the fact that system (34)(35) is identical to the 'plus' Boussinesq equation. We thank Prof. Jeremy Levesley for his help with manuscript preparation. The work has been supported by the Russian Science Foundation (grant no. 14-21-00090).

To the memory of our teachers and friends A. A. Cherepanov, D. V. Lyubimov, and S. V. Shklyaev.
[1] G. H. Wolf, The dynamic stabilization of the RayleighTaylor instability and the corresponding dynamic equilibrium, Z. Phys. 227, 291 (1969).

[2] G. H. Wolf, Dynamic Stabilization of the Interchange Instability of a Liquid-Gas Interface, Phys. Rev. Lett. 24, 444 (1970).

[3] D. V. Lyubimov and A. A. Cherepanov, Development of a steady relief at the interface of fluids in a vibrational field, Fluid Dynamics 21, 849 (1986).

[4] D. V. Lyubimov, M. V. Khenner, and M. M. Shotz, Stability of a Fluid Interface Under Tangential Vibrations, Fluid Dynamics 33, 318 (1998).

[5] M. V. Khenner, D. V. Lyubimov, T. S. Belozerova, and B. Roux, Stability of Plane-Parallel Vibrational Flow in a Two-Layer System, European Journal of Mechanics B/fluids 18, 1085 (1999).

[6] A. V. Zamaraev, D. V. Lyubimov, and A. A. Cherepanov, On equlibrium shapes of the interface between two fluids in vibrational field, in Hydrodynamics and Processes of Heat and Mass Transfer (Ural Branch of Acad. of Science of USSR, Sverdlovsk, 1989), pp. 23-26. [In Russian. Since the translation of this paper into English in not available in the literature, it may be suitable to notice that the results of this work related to the problem we consider can be as well deduced from [3].]
[7] S. V. Shklyaev, A. A. Alabuzhev, and M. Khenner, Influence of a longitudinal and tilted vibration on stability and dewetting of a liquid film, Phys. Rev. E 79, 051603 (2009).

[8] E. S. Benilov and M. Chugunova, Waves in liquid films on vibrating substrates, Phys. Rev. E 81, 036302 (2010).

[9] U. Thiele, J. M. Vega, and E. Knobloch, Long-wave Marangoni instability with vibration, J. Fluid Mech. 546, 61 (2006).

[10] A. A. Nepomnyashchy and I. B. Simanovskii, The influence of vibration on marangoni waves in two-layer films, J. Fluid Mech. 726, 476 (2013).

[11] D. S. Goldobin and D. V. Lyubimov, Soret-Driven Convection of Binary Mixture in a Horizontal Porous Layer in the Presence of a Heat or Concentration Source, JETP 104, 830 (2007).

[12] E. J. Watson, The radial spread of a liquid jet over a horizontal plane, J. Fluid Mech. 20, 481 (1964).

[13] J. Boussinesq, Théorie des ondes et des remous qui se propagent le long d'un canal rectangulaire horizontal, en communiquant au liquide contenu dans ce canal des vitesses sensiblement pareilles de la surface au fond, Journal de Mathématiques Pures et Appliquées 17, 55 (1872).

[14] W. Choi and R. Camassa, Fully nonlinear internal waves 
in a two-fluid system, J. Fluid Mech. 396, 1 (1999).

[15] V. S. Manoranjan, A. R. Mitchell, and J. L. Morris, Numerical solutions of the good Boussinesq equation, SIAM J. Sci. Statist. Comput. 5(4), 946 (1984).

[16] V. S. Manoranjan, T. Ortega, and J. M. Sanz-Serna, Soliton and antisoliton interactions in the "good" Boussinesq equation, J. Math. Phys. 29(9), 1964 (1988).

[17] L. V. Bogdanov and V. E. Zakharov, The Boussinesq equation revisited, Physica D 165, 137 (2002).

[18] J. L. Bona and R. L. Sachs, Global existence of smooth solutions and stability of solitary waves for a generalized Boussinesq equation, Comm. Math. Phys. 118, 15 (1988).

[19] Y. Liu, Instability of solitary waves for generalized Boussinesq equations, J. Dynam. Differential Equations 5, 537 (1993).

[20] T. R. Akylas, Envelope solitons with stationary crests, Phys. Fluids 5, 789 (1993).

[21] M. S. Longuet-Higgins, Capillary-gravity waves of solitary type and envelope solitons on deep water, J. Fluid Mech. 252, 703 (1993).
[22] D. S. Goldobin, K. V. Kovalevskaya, and D. V. Lyubimov, Elastic and inelastic collisions of interfacial solitons and integrability of a two-layer fluid system subject to horizontal vibrations, EPL (Europhys. Lett.) 108, 54001 (2014).

[23] G. El and A. Kamchatnov, Kinetic Equation for a Dense Soliton Gas, Phys. Rev. Lett. 95, 204101 (2005).

[24] In the idealistic dissipation-free case, when there are no attracting states, the basins of attraction are replaced with the basins of dynamics around corresponding states; i.e., of running perturbations around the flat-interface state and of evolving finite-amplitude patterns.

[25] For problems considered in [3 6], moderate discrepancies between two thicknesses resulted only in quantitative corrections.

[26] The fact, that unperturbed unstable analytical solution used as initial conditions could persist for up to 100 time units, suggests the discretisation and numerical integration schemes introduce quite a small inaccuracy into the system simulation. 